\title{
Introducing medical genetics services in Ethiopia using the MiGene Family History App
}

\author{
Shane C. Quinonez, MD ${ }^{1}$, Abate Yeshidinber, MD², Michael A. Lourie, MPH ${ }^{3}$, \\ Delayehu Bekele, MD, $\mathrm{MPH}^{4}$, Yemisrach Mekonnen, $\mathrm{MD}^{2}$, Balkachew Nigatu, $\mathrm{MD}^{4}$, \\ Gesit Metaferia, $\mathrm{MD}^{2}$ and Solomie Jebessa, $\mathrm{MD}^{2}$
}

\begin{abstract}
Purpose: Almost all low-income countries and many middleincome countries lack the capacity to deliver medical genetics services. We developed the MiGene Family History App (MFHA), which assists doctors with family history collection and populationlevel epidemiologic analysis. The MFHA was studied at St. Paul's Hospital in Addis Ababa, Ethiopia.
\end{abstract}

Methods: A needs assessment was used to assess Ethiopian physicians' experience with genetics services. The MFHA then collected patient data over a 6-month period.

Results: The majority of doctors provide genetics services, with only $16 \%$ reporting their genetics knowledge is sufficient. A total of 1699 patients from the pediatric ward $(n=367)$, neonatal intensive care unit (NICU) $(n=477)$, and antenatal clinic $(n=855)$ were collected using the MFHA with a $4 \%$ incidence of a MFHA- screened condition present. The incidence was $11.7 \%$ in the pediatric ward, $3 \%$ in the NICU, and $0.5 \%$ in the antenatal clinic. Heart malformations (5.5\% of patients) and trisomy 21 ( $4.4 \%$ of patients) were the most common conditions in the pediatric ward.

Conclusion: Medical genetics services are needed in Ethiopia. As other countries increase their genetics capacity, the MFHA can provide fundamental genetics services and collect necessary epidemiologic data.

Genetics in Medicine (2019) 21:451-458; https://doi.org/10.1038/s41436018-0069-6

Keywords: Global health; Noncommunicable diseases; Community genetics; Family health history; Mobile health

\section{INTRODUCTION}

Over the last two decades, noncommunicable diseases (NCDs) have steadily increased as causes of worldwide disability and mortality with a concomitant decrease in disease burden from communicable, maternal, neonatal, and nutritional conditions. ${ }^{1}$ This epidemiologic transition has especially affected low and middle-income countries (LMICs), which are responsible for $80 \%$ of all NCD deaths and the majority of worldwide disability-adjusted life years. ${ }^{2,3}$ Major causes of NCDs affecting the adult-aged population include cardiovascular disease, cancer, type 2 diabetes, and others. The most common cause of pediatric NCDs are congenital anomalies with a genetic or partially genetic origin, which affect 7.9 million children worldwide. ${ }^{4}$ LMICs also bear the majority of this pediatric NCD disease burden as they are responsible for $94 \%$ of all births affected with a congenital anomaly. ${ }^{5-7}$ Importantly, the genetic contribution to disease is not limited to pediatric NCDs as genetic factors play an important role in the development and progression of adult-onset NCDs as well. ${ }^{8}$ As the public health impact of NCDs continues to increase in all age groups, equipping LMICs with the medical genetics capacity necessary to diagnosis, treat, and prevent NCDs will become increasingly important. ${ }^{7,9,10}$

For decades, medical genetics-based strategies aimed at addressing pediatric and adult-onset NCDs have been in place in nearly all high-income countries. These strategies, such as prenatal diagnostic services, carrier screening programs, and newborn screening programs, were the direct result of epidemiologic transitions experienced in these countries near-identical to those currently occurring in many LMICs. ${ }^{7}$ Unfortunately, nearly all low-income countries and many middle-income countries lack the necessary personnel, capital, technology, infrastructure, and public and medical education capabilities needed to implement identical highincome country approaches. ${ }^{11}$ To address this gap, community genetics has been proposed as a model that can assist LMICs with the introduction of medical genetics services. ${ }^{11}$ Community genetics aims to prevent congenital anomalies and genetic disease at the population level while simultaneously providing genetics services to affected individuals and their families. ${ }^{11}$ Examples of successfully implemented programs include those targeting sickle cell disease in Cameroon, Jamaica, Tanzania, and Nigeria and those

\footnotetext{
${ }^{1}$ Department of Pediatrics and Communicable Diseases, Division of Genetics, Metabolism and Genomic Medicine, University of Michigan, Ann Arbor, Michigan, USA; ${ }^{2}$ Department of Pediatrics and Child Health, St. Paul's Hospital Millennium Medical College, Addis Ababa, Ethiopia; ${ }^{3}$ University of Michigan Medical School, Ann Arbor, Michigan, USA; ${ }^{4}$ Department of Obstetrics and Gynecology, St. Paul's Hospital Millennium Medical College, Addis Ababa, Ethiopia.

Correspondence: Shane C. Quinonez (squinon@med.umich.edu)
} 
addressing thalassemias in Bahrain, Iran, and Cyprus. ${ }^{12-18}$ Each country has a unique set of disorders and cultural and religious backgrounds and beliefs all requiring consideration prior to program implementation. ${ }^{7}$ Resultantly, genetic services must accommodate the available workforce, align with a country's needs and culture, limit costs associated with any technology, and target sources of genetics information that are both valuable and reasonably attainable. ${ }^{11}$ Community-based genetics programs should target primary healthcare workers and focus on fundamental and inexpensive services most appropriate for their patient population. ${ }^{7}$ One such opportunity is the use of a family history in patient visits-a technique well within the capabilities of LMIC primary healthcare providers. ${ }^{7,11}$

Here we report on the use of a mobile health (mHealth) application, the MiGene Family History App (MFHA), as a means of introducing community genetics services into low-income countries. The MFHA is an Android-based app used by healthcare providers that assists with the collection of patients' family histories, the delivery of genetic counseling, and analysis of the epidemiology of disease affecting a population. The MFHA was launched in 2016 at St. Paul's Hospital Millennium Medical College (SPHMMC), the second largest public hospital in Ethiopia. We report on the app development process, its first 6 months of regular use, and user feedback. We additionally assessed Ethiopian physicians' experiences and comfort with medical genetics principles and practice. To our knowledge this is the first community genetics program in Ethiopia and the first to use a mobile health
(mHealth) tool to introduce medical genetics services into a low-income country.

\section{MATERIALS AND METHODS}

\section{Needs assessment survey design}

A survey was created aimed at assessing Ethiopian physicians' education in genetics and genetic disease and their clinical experience and comfort with family history collection, diagnosis of a suspected genetic disorder, and delivery of genetic counseling. The survey was designed to cover a wide range of topics in efforts to support the MFHA's utility while also providing needs assessment data for future medical genetics capacity building strategies. The survey consisted of two multiple choice questions and one free-text box covering medical genetics training and 19 5-point Likert scale questions, one multiple choice question and two free-text boxes covering practice patterns and comfort levels with medical genetics services (Supplementary Material).

\section{MFHA design and programming}

The MFHA was designed by a study author (SQ) in consultation with SPHMMC study members and programmed by xHub, a technology group in Ethiopia. To optimize use in LMICs, the MFHA was designed for use on Android operating systems, the most commonly used operating system in Ethiopia and worldwide. The MFHA requires no Internet or wireless connection for the majority of its functions as this is often absent in many LMIC healthcare settings. The MFHA has three functions: (1) collection of a patient's family history, (2) delivery of personalized genetic counseling resources based

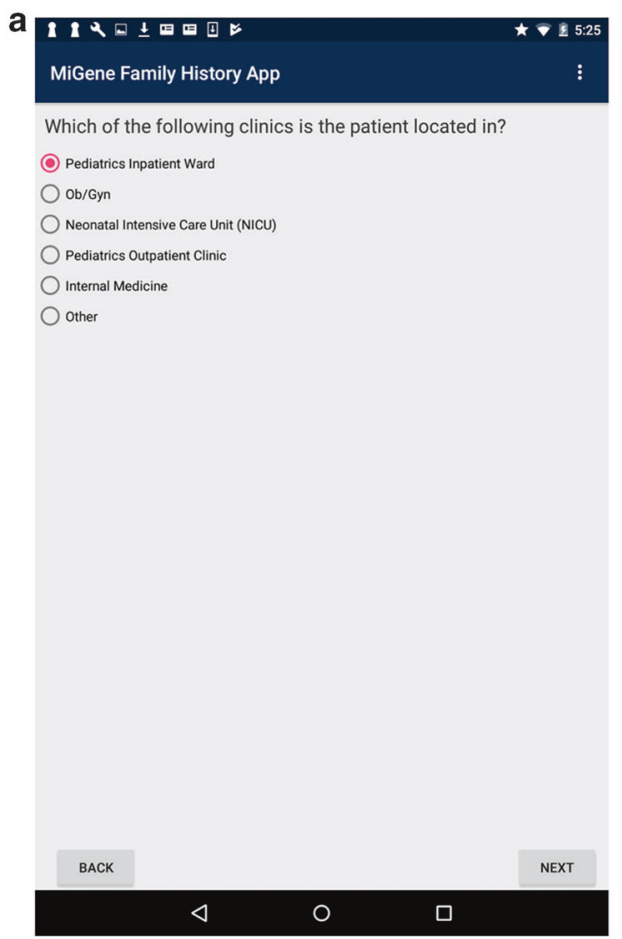

b Trisomy 21 (Down Syndrome)

What is Down syndrome?

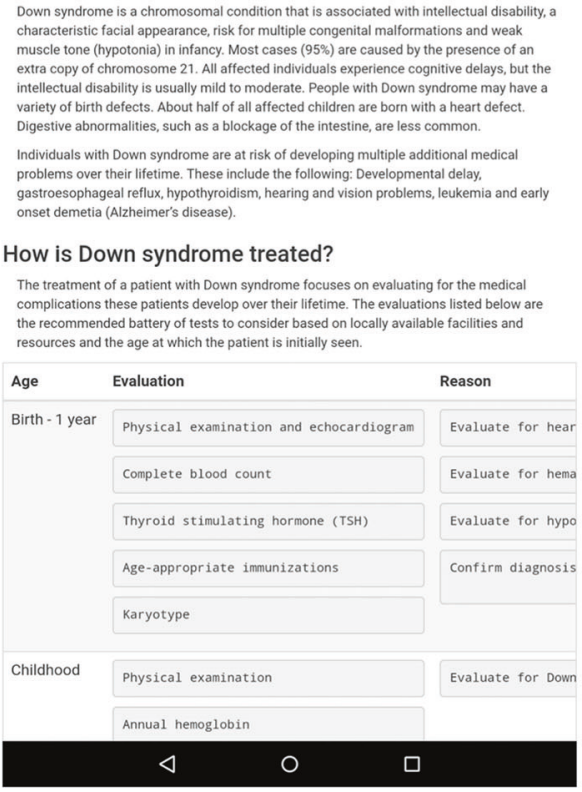

Fig. 1 MiGene Family History App screenshots. a Clinical location entry screen. b Trisomy 21 genetic counseling information 
on entered patient and family history data, (3) analysis of collected data to determine the incidence of congenital anomalies and genetic diseases in a population (Fig. 1). Users are guided through a series of 26 questions, which provides the information necessary to construct a three-generation pedigree. The MFHA focuses on the most common congenital anomalies and genetic diseases worldwide: neural tube defects, trisomy 21, cleft lip and/or cleft palate, heart malformations, hemoglobinopathies, intellectual disability, multiple congenital anomalies, and other single congenital anomaly. ${ }^{4}$ The family history information includes the proband's living and deceased children, siblings, parents, aunts, uncles, and grandparents, and if family members are affected with a congenital anomaly or genetic disease. After data entry, healthcare providers are provided with genetic counseling information on all conditions present in the patient and/or family. The MFHA collects anonymous patient information with only the sex, age, and hospital location recorded. When a wireless or cellular connection is available, stored data can be transmitted via email through a MFHA-created excel sheet containing all MFHA-collected data. A pedigree generated from a patient's MFHA data during the training period of the app's launch is shown in Supplementary Fig. S1. As medicine in Ethiopia is taught and practiced in English, this language is used throughout the MFHA.

In its current state the MFHA does not deliver individualized risk prediction information for each proband, though condition-specific recurrence risk information is provided within each condition's genetic counseling page. For example, in a family with an autosomal dominant form of cleft palate, the MFHA is unable to analyze the pedigree and report a $50 \%$ recurrence risk. For a family with a hemoglobinopathy though, the genetic counseling information provided following family history entry reports a recurrence risk of $25 \%$ for a family affected with sickle cell disease.

\section{Study setting}

SPHMMC is located in Addis Ababa, Ethiopia. Addis Ababa is the capital and largest city of Ethiopia and in 2007 had a population of 2.7 million people. ${ }^{19}$ Ethiopia is a low-income country with a gross domestic product of 72.4 billion US dollars in $2016 .^{20}$ There are 88 individual languages used in Ethiopia with Amharic (29.3\%) and Oromo (33.8\%) being the most common first languages. In Addis Ababa, the most common language is Amharic (71.0\%) with Oromo spoken by $19 \%$ of the population. English is the most common foreign language spoken in Ethiopia and is the primary language of secondary schools and medicine.

SPHMMC primarily serves patients of lower socioeconomic status, providing approximately $75 \%$ of services free of charge. The hospital has 392 beds and admits approximately 300,000 patients annually. The pediatric outpatient department cares for approximately 7000 patients annually, the neonatal intensive care unit (NICU) admits approximately 4000 patients annually, and the pediatric ward admits approximately 1000 patients annually. Little is known on the incidence of congenital anomalies and genetic disease in Ethiopia, though what is known was published in $2014 .^{21}$

To our knowledge, there is one clinical laboratory in Ethiopia offering aneuploidy analysis via multiplex ligationdependent probe analysis, which has only been available for the past 1-2 years. There are currently no clinical geneticists or genetic counselors in Ethiopia.

\section{Physician and patient recruitment}

Early in the project, a Memorandum of Understanding was signed by both institutions. This partnership was strengthened by 4 trips to SPHMMC taken by one of the study's authors (SQ) during the program's creation. Institutional review board (IRB) approval was obtained from the University of Michigan and SPHMMC with an Amharic consent form used. All physicians were provided with inperson training by one study author (SQ) as well as a MFHA User Manual. A final version was loaded onto physicians' tablets in July 2016. To increase patient recruitment, two SPHMMC nurses were also trained and functioned as the major data collectors during the study.

Patients were regularly recruited from the pediatric ward, NICU, and antenatal clinic at SPHMMC between February 2017 and August 2017. Data was entered into the MFHA by the nurse data collectors while interviewing parents of pediatric patients or pregnant women in the antenatal clinic. In the pediatric ward and NICU, pediatric patients were considered probands. The nurse data collectors interviewed parents or patients in either Amharic or Oromo and entered data into the MFHA. The physician users were consulted by nurse data collectors if questions arose regarding a patient's medical history/diagnosis or if questions regarding the study or medical conditions were asked by parents or patients. Patient data was uploaded at least weekly and entered into a secure REDCap database. Participants were eligible for the study if they were admitted to any of the three locations used for the study and ineligible if they had previously participated in the MFHA study. The primary location of recruitment was the pediatric ward. Data was collected Mondays through Fridays.

\section{MFHA validation and user satisfaction}

To determine the validity of MFHA-collected data, 11 standardized three-generation pedigrees were collected by one of the study authors (SQ) and compared with MFHAgenerated data. The author was not present for the family history collected by the MFHA. Data were analyzed for the accuracy of family members and medical conditions present. Pedigrees were scored based on the accuracy of the 27 variables asked by the MFHA. The overall accuracy of appcollected family history data was $89 \%$. When families that did not speak the same language as the data collectors were excluded from analysis, the accuracy increased to $95 \%$. These families often relied on a person in the ward to translate the patient's information to the data collector. This accuracy is higher than that reported by other web-based family history 
tools. ${ }^{22}$ A survey was created assessing user satisfaction and utilized 24 five-item Likert scale questions, five multiple choice questions, and two free-text questions (Supplementary Materials).

\section{MFHA data statistical analysis}

Data retrieved from the MFHA were used to calculate the prevalence of birth defects and genetic diseases among the sample captured during the study period and to compare populations within the study. Following a cross-sectional design, all observations obtained from the pediatric ward, NICU, and antenatal clinic over the study period were included in analyses. Statistical evaluations were calculated based on the presence of conditions in the child in the pediatric ward/NICU or adult woman in the antenatal clinic. Birth defect and genetic disease variables were operationalized dichotomously by whether the condition was present in the patient. All statistical analyses were completed using SAS 9.4.

\section{RESULTS}

\section{Needs assessment survey}

Surveys were distributed to 47 physicians in the Pediatrics and Obstetrics/Gynecology (Ob/Gyn) departments. Twenty-five surveys $(53 \%)$ were returned with the demographics of all respondents shown in Table $\mathbf{1}$. Review of the practice patterns

Table 1 Demographics of surveyed Ethiopian physicians

$$
\text { Variable }
$$

Number

(\%)

\begin{tabular}{|c|c|c|}
\hline \multirow[t]{2}{*}{ Sex } & Male & $17(68)$ \\
\hline & Female & $8(32)$ \\
\hline \multirow[t]{3}{*}{$\mathrm{Age}^{\mathrm{a}}$} & $<30$ years & $12(52)$ \\
\hline & 30-39 years & $8(35)$ \\
\hline & $\geq 40$ years & $3(13)$ \\
\hline \multirow[t]{3}{*}{ Specialty } & Pediatrics & $14(56)$ \\
\hline & Obstetrics/ & $11(44)$ \\
\hline & Gynecology & \\
\hline \multirow[t]{7}{*}{ Medical school ${ }^{a}$} & $\begin{array}{l}\text { University of } \\
\text { Gondor }\end{array}$ & $6(43)$ \\
\hline & Mekelle University & $1(7)$ \\
\hline & SPHMMC & $2(14)$ \\
\hline & Jimma University & $3(21)$ \\
\hline & Hawassa University & $1(7)$ \\
\hline & Addis Ababa & $1(7)$ \\
\hline & University & \\
\hline \multirow[t]{3}{*}{ Residency } & Addis Ababa & $9(36)$ \\
\hline & University & \\
\hline & SPHMMC & $16(64)$ \\
\hline \multirow{4}{*}{$\begin{array}{l}\text { Location(s) of medical genetics } \\
\text { education }^{\text {b }}\end{array}$} & Medical school & $10(67)$ \\
\hline & Residency & $12(80)$ \\
\hline & In current practice & $7(47)$ \\
\hline & Outside resources & $4(27)$ \\
\hline
\end{tabular}

Not all respondents answered these questions

${ }^{b}$ Only attending surveys used for analysis $(n=15)$

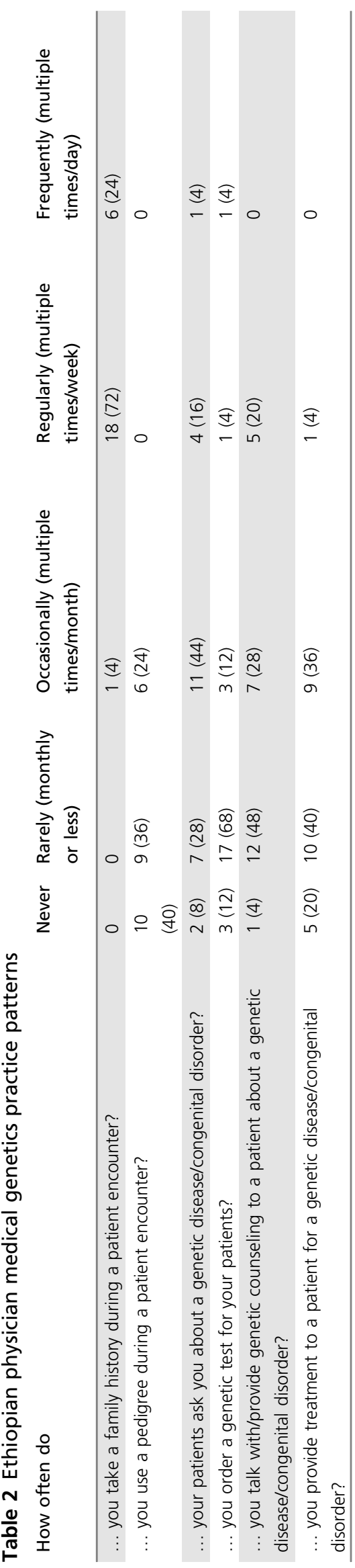

Volume 21 | Number 2 | February 2019| GENETICS in MEDICINE 


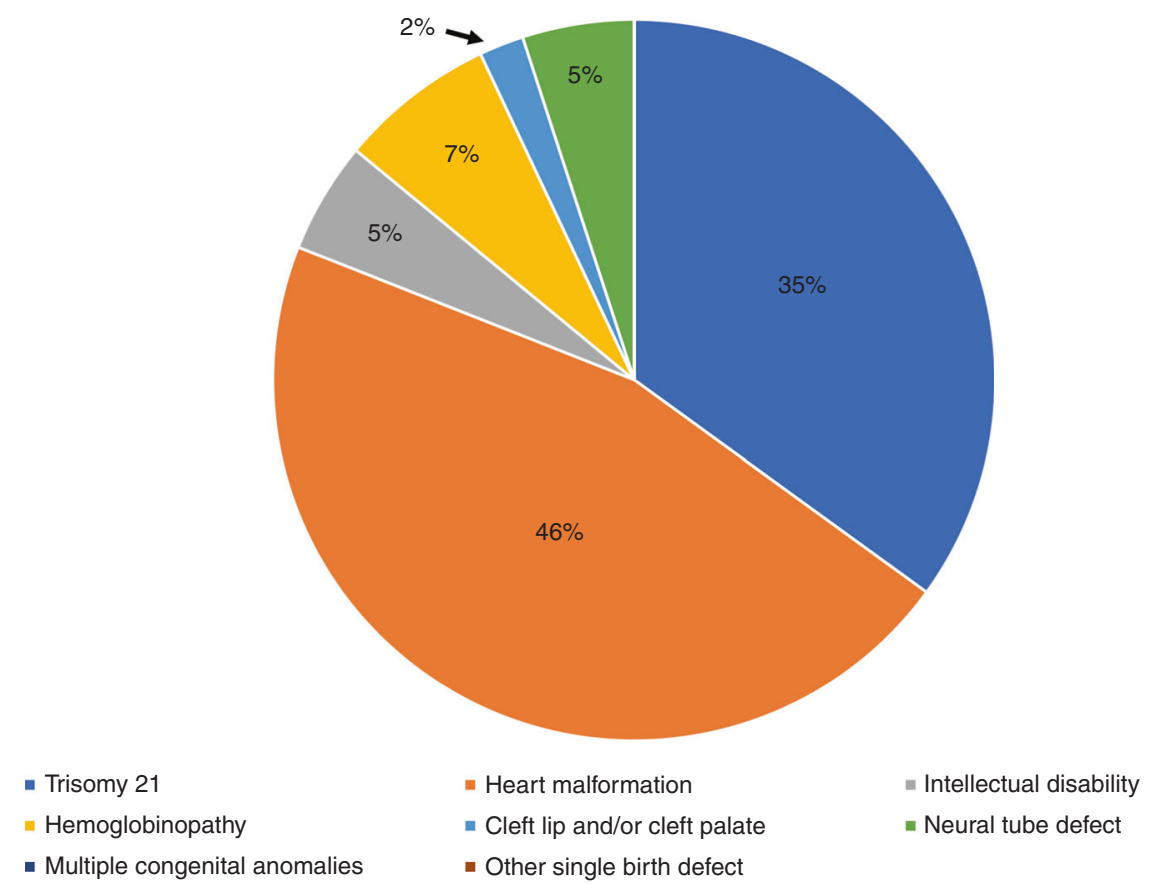

Fig. 2 The incidence of conditions screened for by the MiGene Family History App in the pediatric ward

and confidence level of physicians at SPHMMC is shown in Table 2 and Supplementary Table S1. Of the physicians surveyed, most providers $(24 / 25,96 \%)$ report obtaining a family history when caring for patients, though the majority $(19 / 25,76 \%)$ do not use a pedigree. Most respondents $(19 / 25,76 \%)$ were confident in their ability to collect a family history, but only $6 / 25$ (24\%) were confident in their ability to produce/interpret a pedigree. Over half of surveyed providers $(16 / 25,64 \%)$ report being asked at least multiple times/month about a congenital disorder/genetic disease, with only $7 / 25$ (28\%) rarely asked (monthly or less) about a congenital disorder/genetic disease, and 2/25 (8\%) never asked about a congenital disorder/genetic disease by patients/families. Nearly half of respondents $(12 / 25,48 \%)$ report occasionally, regularly, or frequently providing genetic counseling with 13/ 25 (52\%) reporting rarely or never providing genetic counseling. Only 7/25 (28\%) of respondents felt confident in their ability to counsel/treat a patient based on their family history with the remainder $(18 / 25,72 \%)$ either neutral or not confident in their abilities. When counseling/treating patients based on a pedigree, only $3 / 25(12 \%)$ of respondents reported being confident in their abilities with the majority $16 / 25$ (64\%) not confident. Regarding the ordering of genetic testing, most physicians $(20 / 25,80 \%)$ report ordering testing rarely or never. Providers ordering testing much more often were obstetricians likely recommending prenatal testing for fetuses with multiple congenital anomalies and/or findings concerning for aneuploidy. Overall, only 4/25 (16\%) felt their genetics knowledge was sufficient for their practice with the majority $(18 / 25,72 \%)$ interested in furthering their genetics education.

\section{MFHA-collected SPHMMC data}

During the 6 months of the study, a total of 1699 patients were enrolled from the pediatric ward $(n=367)$, NICU $(n=477)$, and the antenatal clinic $(n=855)$. In the pediatric ward and NICU, this represented 70 and $23 \%$ of all patients admitted during that time, respectively. The low percentage of NICU patients is likely due to the fact that parents are often not at the NICU bedside. These numbers were obtained with one data collector working for 4 months and a second data collector added for months 5 and 6 . The incidence of a MFHA-screened condition in the proband was $4 \%$ $(n=63)$ for the entire population studied. In the pediatric ward the incidence of a condition was $11.7 \%(n=43)$, in the NICU it was $3 \%(n=16)$, and in the antenatal clinic it was $0.5 \%(n=4)$.

Incidence data was most reliable from the pediatric ward as it was the main focus of the study and the location where the highest percentage of admissions were captured. The most common conditions seen in the pediatric ward were heart malformations ( $n=21,5.5 \%$ of all admissions) and trisomy 21 ( $n=16,4.4 \%$ of all admissions). The incidence of other conditions is shown in Fig. 2. Of those patients with a MFHAscreened condition in the pediatric ward, 16/43 (37\%) were male with 27/43 (63\%) female. Of all males and females in the study, $7.8 \%$ of males were affected with a MFHA-screened condition while $16.7 \%$ of females were affected with a MFHAscreened condition. Comparison of these groups found a statistically significant difference in the incidence of a condition in females as compared to males $(p=<0.0088)$. The average age of all patients in the pediatric ward was 2.3 years. No statistical difference $(p=0.8818)$ was seen between 
the average age of patients with a MFHA-screened condition and those without a MFHA-screened condition. Two patients had multiple conditions, the first a 2-year-old girl with Trisomy 21 and a heart malformation and the second a 3year-old boy with multiple congenital anomalies and intellectual disability. During the study period, no families were identified with a positive family history of a congenital anomaly or genetic disease.

In the NICU a total of 16 patients (3\%) were identified with a MFHA-screened condition. One patient had multiple congenital anomalies, 3 had neural tube defects, 1 had trisomy 21,8 had a heart malformation, 2 had intellectual disability, and 1 had a single congenital anomaly not otherwise specified. No clinical data is available to determine the reason "intellectual disability" was selected for a patient in the NICU though it may have been due to hypoxic ischemic encephalopathy. In the antenatal clinic, four patients were affected with a condition; two with a heart malformation and two with intellectual disability. Nine percent of women were found to be of advanced maternal age ( $\geq 35$ years of age).

\section{User feedback}

A total of seven surveys were returned by users and reviewed for feedback. The app was initially distributed to 12 users but primarily used by the seven users surveyed over the study period. User feedback is shown in Table 3 and Supplementary Tables S2 and S3. All users $(7 / 7 ; 100 \%)$ reported the app took 10 minutes or less to complete. Additionally, all users reported "Time constraints" were a contributor to the times they did not use the app, with 5/7 users (71\%) reporting "Time constraints" as the most important factor when choosing to not use the app. All users reported being either very satisfied $(3 / 7 ; 43 \%)$ or somewhat satisfied $(4 / 7 ; 57 \%)$ with the MFHA.

\section{DISCUSSION}

Here we report on the establishment of the first community genetics program in Ethiopia, which utilized a mHealth app for the majority of its services. We detail the program's design process including the initial needs assessment and the programming and launch of the MFHA. The MFHA provided SPHMMC for the first time with prevalence data on congenital anomalies and genetic disease affecting its patient population. This work shows the feasibility and benefit of the expansion of medical genetics services into LMICs and the potential for mHealth technology to assist with this expansion.

Approximately $12 \%$ of all admitted patients in the pediatric ward were affected with a congenital anomaly and/or genetic disease, with heart malformations and trisomy 21 the most common disorders present. The incidence of congenital anomalies and genetic disease in various pediatric inpatient settings has been studied and typically shows an incidence between 10 and 12\%, though some high-income country tertiary centers report an incidence as high as $34 \% .{ }^{23-31}$ As the diagnostic capabilities for congenital anomalies, such as echocardiograms and ultrasounds, are limited at SPHMMC, the actual prevalence of these conditions is likely higher. According to the March of Dimes global report on birth defects, the five most common serious congenital anomalies with genetic or partially genetic origin are: (1) congenital heart defects; (2) neural tube defects; (3) hemoglobin disorders, thalassemia, and sickle cell disease; (4) trisomy 21; and (5) glucose-6-phosphate dehydrogenase deficiency (G6PD). ${ }^{4}$ In our pediatric ward cohort, congenital heart malformations and trisomy 21 were the most common disorders present, consistent with the March of Dimes statistics and similar to data found in other hospital cohort studies. $^{26-31}$ The absence of neural tube defects in the pediatric ward is expected as many affected children do not survive the newborn period. Though limited, data from the NICU showed neural tube defects to be the second most common condition present. The limited hemoglobin disorders in the pediatric ward are likely due to the relatively low prevalence of sickle cell disease in Ethiopia as well as limited in-country diagnostic testing for other hemoglobinopathies. ${ }^{32}$

At SPHMMC, providers are responsible for providing medical genetics services but lack formal training and comfort with delivering this care, something common in sub-Saharan Africa. ${ }^{33}$ Most surveyed SPHMMC providers report feeling responsible for discussing genetic information with their patients but feel their genetics knowledge is insufficient. Given this gap in education/training and clinical expectations, it is not surprising most physicians surveyed expressed an interest in furthering their medical genetics education. This interest is reflected in the recommendations that the public, policy makers, and healthcare providers receive education in important topics such as consanguinity, prenatal and

Table 3 MiGene Family History App (MFHA) user feedback

\begin{tabular}{llllll} 
& 1 (Not difficult at all) & 2 & 3 & 4 & 5 (Very difficult) \\
\hline Overall use of the MFHA & $5(71)$ & $2(29)$ & 0 & 0 & 0 \\
\hline Entering information into the MFHA & $4(57)$ & $2(29)$ & $1(14)$ & 0 & 0 \\
\hline Understanding the MFHA output & $3(43)$ & $3(43)$ & $1(14)$ & 0 & 0 \\
Communicating with patients based on MFHA questions & $2(29)$ & $1(14)$ & $3(43)$ & $1(14)$ & 0 \\
\hline Discussing MFHA generated results with patients & $3(43)$ & 0 & $4(57)$ & 0 & 0 \\
\hline Finding time to complete the full MFHA program & 0 & $2(29)$ & $1(14)$ & $2(29)$ & $2(29)$ \\
\hline Integrating use of the MFHA into clinical practice & 0 & $1(14)$ & $4(57)$ & $1(14)$ & $1(14)$ \\
\hline
\end{tabular}


newborn screening, detection of signs of genetic conditions, and ethical, legal, and social issues. ${ }^{11}$ The National Coalition for Health Professional Education in Genetics have outlined core competencies necessary for all health professionals. ${ }^{34}$ While these are appropriate topics, they were designed for settings where physicians are able to refer patients to genetics professionals, a service unavailable in almost all low-income countries. The genetics education of the public and healthcare workforce in LMICs is an area in need of further study as these efforts require consideration of local needs, cultures, diseases, and languages. ${ }^{11}$

The work described here has a number of intentional and unintentional limitations. A major limitation of the current version of the MFHA is its inability to provide individual pedigree risk predictions, making collected family history data useful only for epidemiologic study. While future versions of the MFHA may include this function, the currently collected family history data, though biased, still provides useful and novel epidemiologic information. As the risk prediction capabilities of the MFHA and Ethiopian providers increases, it will be important to also assess and increase Ethiopia's ability to deliver condition-specific prevention strategies. It should also be noted no positive family history of a MFHAscreened condition was identified during the study, limiting the utility of the family history's collection. The impact of this on the MFHA's usefulness is uncertain at this point. An increase in the number, location, and demographic diversity of recruited patients may reveal important insights on the incidence and heritability of congenital anomalies and genetic disease in Ethiopia.

To simplify the launch of the app, a limited number of conditions were included in the MFHA. Though a free text option was available, this was not routinely used by data collectors. Additionally, the MFHA does not inquire about consanguinity or half-siblings. The Ethiopian prevalence of these family history variables is unknown but clearly present. ${ }^{21}$ Also, the primary data collectors were nurses who lacked training in medical genetics, hindering their ability to fully assess an individual's medical history. Nurses and doctors additionally lacked the training and comfort with the delivery of the natural history/genetic counseling information delivered by the app. While this aspect of the MFHA was not utilized, we feel this is a strength of our work because no information is known regarding what cultural factors need consideration prior to the introduction of genetic counseling services in Ethiopia. Additional limitations include the small number of users and the MFHA's launch at only one institution, resulting in a very biased sample. Additionally, our cohort was limited to families and probands who spoke Amharic and/or Oromo, with many other languages in use throughout Ethiopia. While these two languages are spoken by most SPHMMC patients, it is possible this prevented certain patient enrollment in the study. Importantly, little is known on the preferences of Ethiopians regarding discussing congenital anomalies and genetic diseases. This may have resulted in a concern for lack of anonymity and limited the parents' or patient's willingness to participate in the study. While these factors limit the data's generalizability, the narrow focus of the project likely increased its chances of success during the early stages. No outcomes were measured during this early phase of the MFHA's launch. Future plans include the addition and study of the effects of genetic counseling when combined with the MFHA. Prior to the inclusion of these services, exploring the local patient preferences and beliefs surrounding genetic counseling will be necessary.

The MFHA is undergoing an update by xHub in early 2018 that will incorporate adult-onset conditions such as cancer, cardiovascular disease, and chronic kidney disease and will include consanguinity and half-siblings as collected variables. The inclusion of adult-onset NCDs is important as the use of individuals' family histories has been shown to result in increased uptake of healthcare recommendations, more than genetic testing-informed risk information, in patients with type 2 diabetes, cancer, and cardiovascular disease. ${ }^{35-38}$

The needs of a comprehensive community genetics program are vast. Our project is the first of many needed steps toward the initiation of a nationwide community genetics program in Ethiopia. The near-endless benefits of genetics continue to be realized in many high-income counties, but have yet to be shared with the populations most in need of their services. With the worldwide increasing burden of pediatric and adult-onset NCDs, the use of genetics-based strategies is essential to aid in their diagnosis, prevention, and management. It is our hope the work described here is not regarded as the only way to introduce medical genetics services into LMICs. Rather, we view our work as one step toward the ultimate goal of geneticsinformed care for all, regardless of where they happen to live.

\section{ELECTRONIC SUPPLEMENTARY MATERIAL}

The online version of this article (https://doi.org/10.1038/s41436018-0069-6) contains supplementary material, which is available to authorized users.

\section{ACKNOWLEDGMENTS}

This work was funded by grants from the University of Michigan Department of Pediatrics including the Intramural Research Funding Program (Benz Birth Defects Research Award) and the Percy and Mary Murphy Children's Research Fund.

\section{DISCLOSURE}

The authors declare no conflicts of interest.

\section{REFERENCES}

1. Murray CJL, Lopez AD. Measuring the global burden of disease. N Engl J Med. 2013;369:448-57.

2. Hunter DJ, Reddy KS. Noncommunicable diseases. $N$ Engl J Med. 2013;369:1336-43.

3. World Health Organization. The global burden of disease: 2004 update. 2008. http://www.who.int/healthinfo/global_burden_disease/2004_report update/en/.Accessed 23 January 2018 
4. March of Dimes. March of Dimes global report on birth defects. January 2006:1-76. https://www.marchofdimes.org/mission/march-of-dimesglobal-report-on-birth-defects.aspx.Accessed 23 January 2018

5. GBD 2015 Child Mortality Collaborators. Global, regional, national, and selected subnational levels of stillbirths, neonatal, infant, and under-5 mortality, 1980-2015: a systematic analysis for the Global Burden of Disease Study 2015. Lancet. 2016;388:1725-74.

6. The Global Burden of Disease Child and Adolescent Health Collaboration, Kassebaum N, Kyu HH, et al. Child and Adolescent Health From 1990 to 2015. JAMA Pediatr. 2017;171:573-20.

7. Christianson A, Modell B. Medical genetics in developing countries. Annu Rev Genom Hum Genet. 2004;5:219-65.

8. Sadee W, Hartmann K, Seweryn M, et al. Missing heritability of common diseases and treatments outside the protein-coding exome. Hum Genet. 2014;133:1199-215.

9. Tekola-Ayele F, Rotimi CN. Translational genomics in low- and middleincome countries: opportunities and challenges. Public Health Genomics. 2015;18:1-6.

10. Kingsmore SF, Lantos JD, Dinwiddie DL. Next-generation community genetics for low- and middle-income countries. Genome Med. 2012:4:25-33.

11. World Health Organization. Community genetics services: report of a WHO consultation on community genetics in low-and middle-income countries. 2011. https://apps.who.int/iris/handle/10665/44532. Accessed 23 January 2018

12. Wonkam A, Tekendo CN, Sama DJ, et al. Initiation of a medical genetics service in sub-Saharan Africa: experience of prenatal diagnosis in Cameroon. Eur J Med Genet. 2011;54:e399-e404.

13. Makani J, Soka D, Rwezaula S, et al. Health policy for sickle cell disease in Africa: experience from Tanzania on interventions to reduce under-five mortality. Trop Med Int Health. 2015;20:184-7.

14. Grosse SD, Atrash HK, Odame I, et al. The Jamaican historical experience of the impact of educational interventions on sickle cell disease child mortality. Am J Prev Med. 2012;42:e101-e103.

15. Galadanci N, Wudil BJ, Balogun TM, et al. Current sickle cell disease management practices in Nigeria. Int Health. 2014;6:23-28.

16. Arrayed AIS. Campaign to control genetic blood diseases in Bahrain. Community Genet. 2005;8:52-55.

17. Samavat A, Modell B. Iranian national thalassaemia screening programme. BMJ. 2004;329:1134-7.

18. Angastiniotis MA, Hadjiminas MG. Prevention of thalassaemia in Cyprus. Lancet. 1981;1:369-71.

19. The World Bank. 2016. Ethiopia_population and housing census 2007-IPUMS subset. http://microdata.worldbank.org/index.php/catalog/ 2747 Accessed 24 February 2018.

20. The World Bank. 2016. Ethiopia. https://data.worldbank.org/country/ ethiopia Accessed 24 February 2018.

21. Tadesse L, Tafesse F, Hamamy H. Communities and community genetics in Ethiopia. Pan Afr Med J. 2014;18:115.
22. Wang $C$, Bickmore $T$, Bowen DJ, et al. Acceptability and feasibility of a virtual counselor (VICKY) to collect family health histories. Genet Med. 2015;17:822-30.

23. McCandless SE, Brunger JW, Cassidy SB. The burden of genetic disease on inpatient care in a children's hospital. Am J Hum Genet. 2004:74:121-7.

24. Walker CE, Mahede T, Davis G, et al. The collective impact of rare diseases in Western Australia: an estimate using a population-based cohort. Genet Med. 2017:19:546-52.

25. Colvin L, Bower C. A retrospective population-based study of childhood hospital admissions with record linkage to a birth defects registry. $B M C$ Pediatr. 2009;9:32.

26. Hall JG, Powers EK, Mcllvaine RT, et al. The frequency and financial burden of genetic disease in a pediatric hospital. Am J Med Genet. 1978;1:417-36.

27. Carnevale A, Hernández $M$, Reyes $R$, et al. The frequency and economic burden of genetic disease in a pediatric hospital in Mexico City. Am J Med Genet. 1985:20:665-75.

28. FitzPatrick DR, Skeoch CH, Tolmie JL. Genetic aspects of admissions to a paediatric intensive care unit. Arch Dis Child. 1991;66:639-41.

29. Yoon PW, Olney RS, Khoury MJ, et al. Contribution of birth defects and genetic diseases to pediatric hospitalizations. A population-based study. Arch Pediatr Adolesc Med. 1997;151:1096-103.

30. Ling EW, Sosuan LC, Hall JC. Congenital anomalies: an increasingly important cause of mortality and workload in a neonatal intensive care unit. Am J Perinatol. 1991;8:164-9.

31. Scriver CR, Neal JL, Saginur R, et al. The frequency of genetic disease and congenital malformation among patients in a pediatric hospital. Can Med Assoc J. 1973:108:1111-5.

32. Piel FB, Hay SI, Gupta S, et al. Global burden of sickle cell anaemia in children under five, 2010-2050: modelling based on demographics, excess mortality, and interventions. PLoS Med. 2013;10:e1001484.

33. He LQ, Njambi L, Nyamori JM, et al. Developing clinical cancer genetics services in resource-limited countries: the case of retinoblastoma in Kenya. Public Health Genomics. 2014;17:221-7.

34. Core Competency Working Group of the National Coalition for Health Professional Education in Genetics. Recommendations of core competencies in genetics essential for all health professionals. Genet Med. 2001;3:155-9.

35. Ruffin MT, Nease DE, Sen A, et al. Effect of preventive messages tailored to family history on health behaviors: the Family Healthware Impact Trial. Ann Fam Med. 2011:9:3-11.

36. Kahn LB, Marshall JA, Baxter J, et al. Accuracy of reported family history of diabetes mellitus: results from San Luis Valley Diabetes Study. Diabetes Care. 1990;13:796-8

37. Wu RR, Myers RA, Hauser ER, et al. Impact of genetic testing and family health history based risk counseling on behavior change and cognitive precursors for type 2 diabetes. J Genet Couns. 2017;26:133-40.

38. Hariri S, Yoon PW, Qureshi N, et al. Family history of type 2 diabetes: a population-based screening tool for prevention? Genet Med. 2006;8:102-8. 